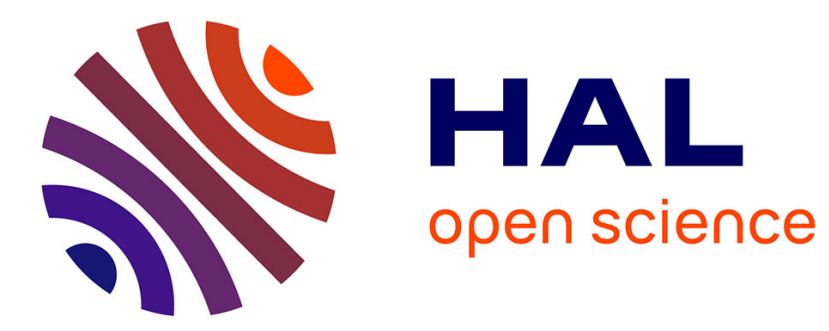

\title{
Stiffness of a wobbling mass models analysed by a smooth orthogonal decomposition of the skin movement relative to the underlying bone
}

Raphaël Dumas, Eric Jacquelin

\section{- To cite this version:}

Raphaël Dumas, Eric Jacquelin. Stiffness of a wobbling mass models analysed by a smooth orthogonal decomposition of the skin movement relative to the underlying bone. Journal of Biomechanics, 2017, 62, pp.47-52. 10.1016/j.jbiomech.2017.06.002 . hal-01635773

\section{HAL Id: hal-01635773 \\ https://hal.science/hal-01635773}

Submitted on 15 Nov 2017

HAL is a multi-disciplinary open access archive for the deposit and dissemination of scientific research documents, whether they are published or not. The documents may come from teaching and research institutions in France or abroad, or from public or private research centers.
L'archive ouverte pluridisciplinaire HAL, est destinée au dépôt et à la diffusion de documents scientifiques de niveau recherche, publiés ou non, émanant des établissements d'enseignement et de recherche français ou étrangers, des laboratoires publics ou privés. 


\section{Accepted Manuscript}

Stiffness of a wobbling mass models analysed by a smooth orthogonal decomposition of the skin movement relative to the underlying bone

Raphaël Dumas, Eric Jacquelin

PII:

S0021-9290(17)30295-6

DOI: http://dx.doi.org/10.1016/j.jbiomech.2017.06.002

Reference: BM 8241

To appear in:

Journal of Biomechanics

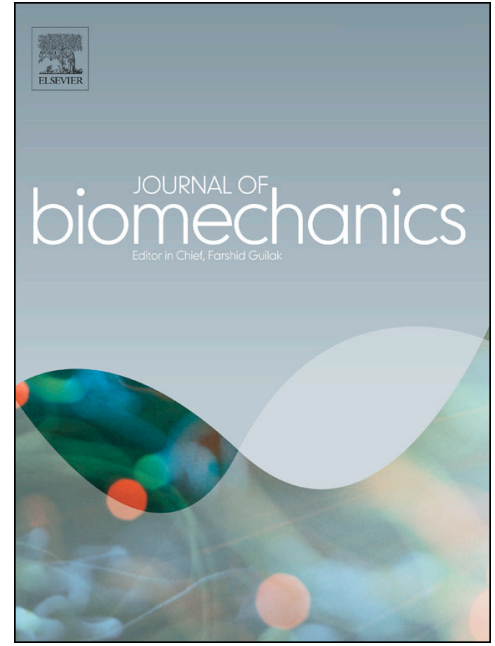

Received Date: $\quad 19$ February 2017

Revised Date: $\quad 17$ May 2017

Accepted Date: $\quad 5$ June 2017

Please cite this article as: R. Dumas, E. Jacquelin, Stiffness of a wobbling mass models analysed by a smooth orthogonal decomposition of the skin movement relative to the underlying bone, Journal of Biomechanics (2017), doi: http://dx.doi.org/10.1016/j.jbiomech.2017.06.002

This is a PDF file of an unedited manuscript that has been accepted for publication. As a service to our customers we are providing this early version of the manuscript. The manuscript will undergo copyediting, typesetting, and review of the resulting proof before it is published in its final form. Please note that during the production process errors may be discovered which could affect the content, and all legal disclaimers that apply to the journal pertain. 
Stiffness of a wobbling mass models analysed by a smooth orthogonal decomposition of the skin movement relative to the underlying bone

Raphaël Dumas $^{1,2}$ \& Eric Jacquelin ${ }^{1}$

${ }^{1}$ Univ Lyon, Université Claude Bernard Lyon 1, IFSTTAR, LBMC UMR_T9406, F69622, Lyon, France

2 Interuniversity Centre of Bioengineering of the Human Neuromusculoskeletal System, Università degli Studi di Roma “Foro Italico”, Rome, Italy

\section{Abstract}

The so-called soft tissue artefacts and wobbling masses have both been widely studied in biomechanics, however most of the time separately, from either a kinematics or a dynamics point of view. As such, the estimation of the stiffness of the springs connecting the wobbling masses to the rigid-body model of the lower limb, based on the in vivo displacements of the skin relative to the underling bone, has not been performed yet. For this estimation, the displacements of the skin markers in the bone-embedded coordinate systems are viewed as a proxy for the wobbling mass movement.

The present study applied a structural vibration analysis method called smooth orthogonal decomposition to estimate this stiffness from retrospective simultaneous measurements of skin and intra-cortical pin markers during running, walking, cutting and hopping.

For the translations about the three axes of the bone-embedded coordinate systems, the estimated stiffness coefficients (i.e. between $2.3 \mathrm{kN} / \mathrm{m}$ and $55.5 \mathrm{kN} / \mathrm{m}$ ) as well as the 
corresponding forces representing the connection between bone and skin (i.e. up to $400 \mathrm{~N}$ ) and corresponding frequencies (i.e. in the band $10-30 \mathrm{~Hz}$ ) were in agreement with the literature. Consistently with the STA descriptions, the estimated stiffness coefficients were found subject- and task-specific.

\section{Keywords:}

Soft tissue motion, marker-cluster translations, vibration modes, covariance matrix, eigenvalues 


\section{Nomenclature (order of appearance)}

$i$ : index for segment

$j$ : index for marker

$k$ : index for sampled instant of time

$n$ : number of sampled instants of time

$m$ : number of markers on a segment

$\mathbf{v}, \mathbf{V}$ : STA vector, STA field

$\mathbf{S}$ : sample covariance matrix

D: differential operator

$f$ : sampling frequency

$\mathbf{U}$ : velocity covariance matrix

$\boldsymbol{\Psi}$ : smooth orthogonal vector

$\lambda, \lambda$ : smooth orthogonal value, diagonal matrix of eigenvalues

$\omega:$ circular frequency

$\mathbf{K}, \tilde{\mathbf{K}}, \tilde{K}$ : stiffness matrix, stiffness coefficient

$M, \mathbf{M}$ : mass, mass matrix

E: identity matrix

$\Phi$ : basis vector (i.e. marker-cluster geometrical transformation)

F: force vector

a: modal amplitude

$\bar{v}:$ displacement of the centroid of the marker-cluster 


\section{Introduction}

The movement of the skin, muscles, and fat relative to the underlying bone is a well-known phenomenon. It has been described, from a kinematics point of view, as the soft tissue artefact (STA). Indeed, this relative movement has a deleterious effect on the joint kinematics estimated from skin markers and motion capture systems (Leardini et al., 2005; Peters et al., 2010). At the same time, from a dynamics point of view, the soft tissue motion, modelled as wobbling masses connected to the rigid-body model of the lower limb, is also recognised to have an effect on the joint kinetics (i.e. energy dissipation, torque reduction) (Challis and Pain, 2008; Gruber et al., 1998) during motor tasks involving impacts with the ground.

One key parameter of these wobbling mass models is the stiffness of the springs connecting them to the rigid-bodies. Most of the models of the literature include linear or non-linear springs attached to a wobbling mass that can translate (and eventually rotate) with respect to the bone (Alonso et al., 2007; Gittoes et al., 2006; Gruber et al., 1998; Günther et al., 2003; McLean et al., 2003; Pain and Challis, 2004; Wilson et al., 2006). Identification of the parameters of these wobbling mass models, based on the ground reaction forces, as well as sensitivity analyses have been widely performed (Alonso et al., 2007; Gittoes et al., 2009; Pain and Challis, 2004; Wilson et al., 2006). However, to the best of the author's knowledge, the estimation of the stiffness parameters from the displacements of the skin relative to the underling bone measured in vivo by intra-cortical pins has not been performed yet. For this estimation, the displacements of the skin markers in the bone-embedded coordinate systems are viewed as a proxy for the wobbling mass movement.

The objective of this study was to estimate the stiffness matrix of a wobbling mass model, defined as a cluster of lumped masses undergoing translations about the three axes of the bone-embedded coordinate system, by applying a structural vibration analysis method, called smooth orthogonal decomposition (Chelidze and Zhou, 2006), to the simultaneous 
measurements of skin and intra-cortical pin markers (Benoit et al., 2006; Reinschmidt et al., 1997). In this method, the displacement of the skin markers relative to the underlying bone was modelled as the free undamped vibrations of a dynamical system for which the stiffness matrix can be straightforwardly identified.

\section{Material and methods}

Smooth orthogonal decomposition of the skin movement relative to the underlying bone

The STA vector, $\mathbf{v}_{i}^{j}(k)$, was defined to represent the displacement that the skin marker $j$ $\left(j=1: m_{i}\right)$ associated with the segment $i(i=1$ for shank and $i=2$ for thigh) underwent relative to a relevant bone-embedded coordinate system and a reference position at each discrete time $k(k=1: n)$ during the analysed motor task (Dumas et al., 2014a). The STA of all markers on the segment $i$ were represented using the STA field, $\mathbf{V}_{i}(k)$ :

$$
\mathbf{V}_{i}(k)=\left(\begin{array}{c}
\vdots \\
\mathbf{v}_{i}^{j}(k) \\
\vdots
\end{array}\right)
$$

A sample covariance matrix was computed from this STA field known at every sampled instants of time:

$$
\mathbf{S}_{i}=\frac{1}{n}\left[\begin{array}{lll}
\cdots & \mathbf{V}_{i}(k) & \cdots
\end{array}\right]\left[\begin{array}{lll}
\cdots & \mathbf{V}_{i}(k) & \cdots
\end{array}\right]^{T}
$$

This sample covariance matrix has been used in previous studies to compute, by a proper orthogonal decomposition, the main components of the STA during a running task (Dumas et al., 2014a, b). In the smooth orthogonal decomposition, a differential operator was further introduced:

$$
\mathbf{D}=f\left[\begin{array}{ccccc}
-1 & 1 & 0 & \cdots & 0 \\
0 & -1 & 1 & \cdots & 0 \\
\vdots & \ddots & \ddots & \ddots & 0 \\
0 & \cdots & 0 & -1 & 1
\end{array}\right]
$$


where $f$ was the sampling frequency.

This differential operator allowed the computation of the other covariance matrix standing for the velocities of the skin markers:

$$
\mathbf{U}_{i}=\frac{1}{n-1} \mathbf{D}\left[\begin{array}{lll}
\cdots & \mathbf{V}_{i}(k) & \cdots
\end{array}\right]\left[\begin{array}{lll}
\cdots & \mathbf{V}_{i}(k) & \cdots
\end{array}\right]^{T}\left[\begin{array}{l}
\mathbf{D}
\end{array}\right]^{T}
$$

The smooth orthogonal modes were solutions of the eigen-problem:

$\mathbf{S}_{i}\left[\begin{array}{lll}\cdots & \boldsymbol{\Psi}_{i}^{l} & \cdots\end{array}\right]=\boldsymbol{\lambda}_{i} \mathbf{U}_{i}\left[\begin{array}{lll}\cdots & \boldsymbol{\Psi}_{i}^{l} & \cdots\end{array}\right]$

with $\Psi_{i}^{l}\left(l=1: 3 m_{i}\right)$ the smooth orthogonal vectors and $\left(\omega_{i}^{l}\right)^{2}=\left(\lambda_{i}^{l}\right)^{-1}$ the circular frequencies related to the smooth orthogonal values. Assuming that the STA field was the results of free undamped vibrations of a cluster of lumped masses (i.e. each markers having a same mass $\left.M_{i} / m_{i}\right)$, the smooth orthogonal modes are good estimates of the linear normal modes. Therefore, the dynamics of the marker-cluster, as observed from the STA field, was characterized by:

$$
\mathbf{K}_{i}\left[\begin{array}{lll}
\cdots & \boldsymbol{\Psi}_{i}^{l} & \cdots
\end{array}\right]=\mathbf{M}_{i}\left[\begin{array}{lll}
\cdots & \boldsymbol{\Psi}_{i}^{l} & \cdots
\end{array}\right]\left[\boldsymbol{\lambda}_{i}\right]^{-1}
$$

with $\mathbf{K}_{i}$ the stiffness matrix, $\lambda_{i}$ a diagonal matrix composed of the smooth orthogonal eigenvalues and $\mathbf{M}_{i}=\frac{M_{i}}{m_{i}} \mathbf{E}$ the mass matrix (i.e. with $\mathbf{E}$ the identity matrix of dimension $\left.3 m_{i} \times 3 m_{i}\right)$

Therefore, the stiffness matrix was given by:

$$
\mathbf{K}_{i}=\frac{M_{i}}{m_{i}}\left[\begin{array}{lll}
\cdots & \Psi_{i}^{l} & \cdots
\end{array}\right]\left[\lambda_{i}\right]^{-1}\left[\begin{array}{lll}
\cdots & \Psi_{i}^{l} & \cdots
\end{array}\right]^{-1}
$$

According to the recent descriptions of the STA (Andersen et al., 2012; Benoit et al., 2015; Dumas et al., 2015; Grimpampi et al., 2014) and to the wobbling mass models reported in the literature (Alonso et al., 2007; Bélaise et al., 2016; Challis and Pain, 2008; Gittoes et al., 2009; Gruber et al., 1998; Günther et al., 2003; McLean et al., 2003; Wilson et al., 2006), it 
was useful to retrieve the stiffness matrix corresponding only to the modes defining the marker-cluster geometrical rigid transformations and more specifically to the marker-cluster translations. This stiffness matrix was given by:

$$
\tilde{\mathbf{K}}_{i}=\left[\begin{array}{lll}
\cdots & \boldsymbol{\Phi}_{i}^{l} & \cdots
\end{array}\right]^{T} \mathbf{K}_{i}\left[\begin{array}{lll}
\cdots & \boldsymbol{\Phi}_{i}^{l} & \cdots
\end{array}\right]
$$

where $\boldsymbol{\Phi}_{i}^{l}(l=1: 3)$ were the unitary basis vectors built a priori (Dumas et al., 2014a) to define the 3 translations of the marker-cluster about the axes of the bone-embedded coordinate system. These basis vectors were:

$$
\boldsymbol{\Phi}_{i}^{1}=\left(\begin{array}{c}
\vdots \\
1 / \sqrt{m_{i}} \\
0 \\
0 \\
\vdots
\end{array}\right), \boldsymbol{\Phi}_{i}^{2}=\left(\begin{array}{c}
\vdots \\
0 \\
1 / \sqrt{m_{i}} \\
0 \\
\vdots
\end{array}\right) \text {, and } \boldsymbol{\Phi}_{i}^{3}=\left(\begin{array}{c}
\vdots \\
0 \\
0 \\
1 / \sqrt{m_{i}} \\
\vdots
\end{array}\right)
$$

Finally, the force vector representing the connection between the bone and skin, applied to each marker $j$ at each discrete time $k$ along each axes of the bone-embedded coordinate system, were given by:

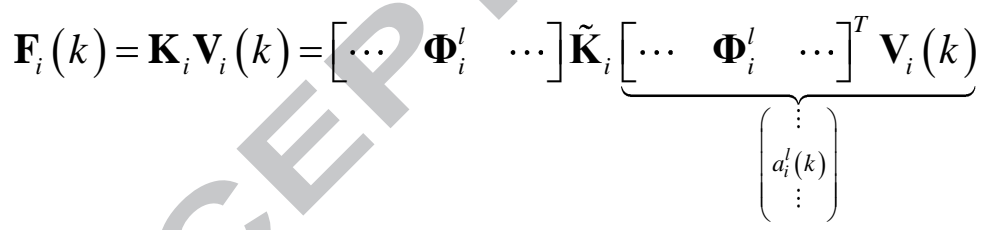

Note that the last factors of Eq.10 matched the definition of the amplitude of the STA (Dumas et al., 2014a), that is to say the projection of the STA field on a given mode:

$$
a_{i}^{l}(k)=\left(\boldsymbol{\Phi}_{i}^{l}\right)^{T} \mathbf{V}_{i}(k)
$$

As the basis vectors $\Phi_{i}^{l}(l=1: 3)$ represented the translations of the marker-cluster, each marker $j$ had the same projected displacement and, therefore, the same force. Moreover, $\bar{v}_{i}^{l}(k)=\frac{a_{i}^{l}(k)}{\sqrt{m_{i}}}$ directly corresponded to the projected displacement of the centroid of the 
marker-cluster about the relevant axis of the bone-embedded coordinate system (i.e. $l=1$ for $\mathrm{X}$ axis, $l=2$ for $\mathrm{Y}$ axis, and $l=3$ for $\mathrm{Z}$ axis). The basis vectors $\boldsymbol{\Phi}_{i}^{l}(l=1: 3)$ were also orthogonal and, therefore, the stiffness matrix defining the marker-cluster translations was $\operatorname{diagonal}\left(\right.$ i.e. $\tilde{\mathbf{K}}_{i}=\operatorname{diag}\left(\begin{array}{lll}\cdots & \tilde{K}_{i}^{l} & \cdots\end{array}\right)$ ).

\section{Experimental data}

The retrospective data used in this study included the right thigh and shank movements from five trials of a running task (i.e. stance phase, from ground contact to take-off) performed by three able-bodied male subjects (Reinschmidt et al., 1997), and from one trial of walking and cutting tasks (i.e. stance phase, from ground contact to take-off) and hopping task (i.e. during $0.67 \mathrm{~s}$ after ground contact) performed by one able-bodied male subject (Benoit et al., 2006). Clusters of markers were attached to intra-cortical pins inserted into the lateral tibial and femoral epicondyles. Both intra-cortical pin markers and skin markers (i.e. between 4 and 6 by segments) were tracked using either three high-speed cameras at $200 \mathrm{~Hz}$ (Reinschmidt et al., 1997) or tracked using four infrared cameras at $120 \mathrm{~Hz}$ (Benoit et al., 2006). Unfiltered raw data were used. The bone-embedded coordinate systems were defined using radiostereometry analysis (Lafortune et al., 1992), with $\mathrm{X}$ axis defined anterior, $\mathrm{Y}$ axis proximal, and $\mathrm{Z}$ axis lateral.

The subjects' demographics and experimental setup are summarised in Table 1. The wobbling masses $M_{i}$ were estimated with $4.8 \%$ and $12.3 \%$ of the total body mass (Dumas et al., 2007) and $77.65 \%$ and $90.3 \%$ (i.e. skin, adipose tissue, and muscle (Clarys and Marfell-Jones, 1986)) of the segment mass for the shank and thigh, respectively.

\section{Results}


Figure 1 represents the stiffness coefficients (i.e. median, quartiles, minimum and maximum for the five running trials of subjects R1, R2 and R3) for the translation of the maker-cluster about the $\mathrm{X}, \mathrm{Y}$ and $\mathrm{Z}$ axes of the bone-embedded coordinate systems for the different subjects and motor tasks. The stiffness appeared generally higher for the shank (i.e between 10.2 $\mathrm{kN} / \mathrm{m}$ and $55.5 \mathrm{kN} / \mathrm{m}$ ) than for the thigh (i.e. between $2.3 \mathrm{kN} / \mathrm{m}$ and $11.1 \mathrm{kN} / \mathrm{m}$ ). The stiffness was higher about the $\mathrm{X}$ axis than about the $\mathrm{Y}$ and $\mathrm{Z}$ axes. The stiffness was also higher for the three subjects (R1, R2, and R3) performing the running task than for the subject (R1) performing walking, cutting and hopping tasks, except for the thigh during the cutting and hopping tasks.

Figure 2 illustrates the forces (i.e. median for the five running trials of subjects R1, R2 and R3) representing the connection between the bone and skin masses about the $\mathrm{X}, \mathrm{Y}$, and $\mathrm{Z}$ axes of the bone-embedded coordinate systems for the different subjects and motor tasks. The forces were generally two times higher for the shank (i.e. up to $400 \mathrm{~N}$ during running) than for the thigh (i.e. up to $100 \mathrm{~N}$ during running). The forces were also higher for the three subjects (R1, R2, and R3) performing the running task than for the subject (R1) performing walking, cutting and hopping tasks. The forces were mainly about the $\mathrm{X}$ and $\mathrm{Y}$ axes, but remarkably about the $\mathrm{X}$ axis of the tibia-embedded coordinate systems during the hopping task. Some similarities in the pattern of the forces during the running tasks could be observed.

\section{Discussion}

This study applied a structural vibration analysis method called smooth orthogonal decomposition to estimate the stiffness matrix of a wobbling mass model from simultaneous measurements of skin and intra-cortical pin markers. The wobbling mass model consisted of a cluster of lumped masses (or, equivalently, of a concentrated mass at the cluster centroid) 
undergoing translations about the three axes of the bone-embedded coordinate system. Yet, Eqs. 8 and 10, allows to define stiffness matrix $\tilde{\mathbf{K}}_{i}$, and force vector $\mathbf{F}_{i}$, corresponding to any basis vectors $\Phi_{i}^{l}$ defining the rigid or non-rigid marker-cluster geometrical transformations or any other change of configuration in the skin marker positions. In this case, the stiffness matrix may not be diagonal and the force applied to each markers of the segment can be different. For instance, in the literature, rotations about the axes of the bone-embedded coordinate system have been also considered in some wobbling mass models (Gruber et al., 1998; Schmitt and Günther, 2011). Other possible changes of configuration are the smooth orthogonal vectors presented in this study $\mathbf{\Psi}_{i}^{l}$ as well as the proper orthogonal vectors previously proposed to describe the main components of the STA (Dumas et al., 2014b; Dumas et al., 2015). Though, the smooth orthogonal vectors, together with the circular frequencies $\omega_{i}^{l}$, are the only estimates which are fully representative of the linear normal modes of the wobbling mass model. Thus, the stiffness matrix $\mathbf{K}_{i}$ can be determined through an appropriate eigen-decomposition where the inverse of the eigenvalues $\left[\boldsymbol{\lambda}_{i}\right]^{-1}$ are associated to the natural frequencies (Chelidze and Zhou, 2006). When having determined the stiffness matrix $\tilde{\mathbf{K}}$ corresponding to the translations of the marker-cluser, the introduction of the amplitude (i.e. with $\bar{v}_{i}^{l}=\frac{a_{i}^{l}}{\sqrt{m_{i}}}$ being the translation of the centroid of the marker-cluster) is useful to implement the forces representing the connection between bone and skin in a dynamic model. These can be representative of the forces between the rigid-body and the wobbling mass to be introduced in the inverse dynamic computation (Alonso et al., 2007; Günther et al., 2003). In the practical case, without the measurements of intra-cortical pin markers, the translation of the centroid of the marker-cluster can be estimated within a multibody kinematics optimisation (Bélaise et al., 2016; Cerveri et al., 2005; Richard et al., 2012). 
From the skin and intra-cortical pin data used in the present study, the estimated values of the stiffness coefficients and forces were found to be different between the different motor tasks. For instance, for the $\mathrm{Y}$ axis of the tibia-embedded coordinate system, the stiffness coefficients were $11.7 \mathrm{kN} / \mathrm{m}$ for walking, $21.4 \mathrm{kN} / \mathrm{m}$ for cutting, $18.4 \mathrm{kN} / \mathrm{m}$ for hopping, and between $32.4 \mathrm{kN} / \mathrm{m}$ and $49.7 \mathrm{kN} / \mathrm{m}$ for running. For the $\mathrm{Y}$ axis of the femur-embedded coordinate system, the stiffness coefficients were $0.4 \mathrm{kN} / \mathrm{m}$ for walking, $0.5 \mathrm{kN} / \mathrm{m}$ for cutting, $0.7 \mathrm{kN} / \mathrm{m}$ for hopping, and between $0.6 \mathrm{kN} / \mathrm{m}$ and $0.9 \mathrm{kN} / \mathrm{m}$ for running. An intra- and inter- variability also appeared across the three subjects performing the running task although some similar patterns of force was observed. As STA is well known to be subject- and task-specific (Leardini et al., 2005; Peters et al., 2010), it was expected to obtain such differences. Moreover, they could be due to the differences in impact forces and muscle activities (however not known in the present study) according to the concept of muscle tuning (Nigg and Wakeling, 2001).

The stiffness coefficients estimated in the present study (Fig. 1) are overall comparable to the other linear parameters reported in the literature to connect the wobbling masses to the rigidbodies. A range from $17.5 \mathrm{kN} / \mathrm{m}$ to $27 \mathrm{kN} / \mathrm{m}$ (Gittoes et al., 2009; Gittoes et al., 2006) for the shank and from $12 \mathrm{kN} / \mathrm{m}$ (Gittoes et al., 2009) to $64 \mathrm{kN} / \mathrm{m}$ (McLean et al., 2003) for the thigh was reported. The forces estimated in the present study (Fig. 2) are also in agreement with the literature. By estimating the acceleration (multiplied by the wobbling mass $M_{i}$ ) of the centroid of the marker-cluster with respect to the position of other skin makers placed at joint level, Schmitt and Günther (2011) reported maximal vertical forces of $409 \mathrm{~N}$ and $684 \mathrm{~N}$ and maximal horizontal forces of $111 \mathrm{~N}$ and $390 \mathrm{~N}$ for the shank and thigh respectively during running. Using accelerometers placed on the muscle belly of tibialis anterior, lateral gastrocnemius, vastus lateralis, and biceps femoris long head, Wakeling et al., (2003) similarly reported vertical forces between $24 \mathrm{~N}$ and $196 \mathrm{~N}$. Moreover, using the scalar 
equation $F_{i}^{l}=M_{i}\left(2 \pi f_{i}^{l}\right)^{2} \bar{v}_{i}^{l}$, Challis and Pain (2008) estimated a maximal vertical force of $315 \mathrm{~N}$ for the shank. Considering the wobbling masses of $2-3 \mathrm{Kg}$ for the shank and 7-10 $\mathrm{Kg}$ for the thigh (Table 1), it can be recognized (i.e. $\left.\left(2 \pi f_{i}^{l}\right)^{2}=\frac{\tilde{K}_{i}^{l}}{M_{i}}\right)$ that the vibration frequencies were in a band 10-30 Hz. This appears in agreement, but in the lower end of the range, with the literature (Lafortune et al., 1995; Pain and Challis, 2006; Schmitt and Günther, 2011; Wakeling et al., 2003).

Using the same walking, cutting, and hopping data from Benoit et al. (2006) as in the present study, Andersen et al. (2012) applied a principal component analysis and demonstrated that the displacements of the skin markers relative to the underling bone can be represented by a linear combination of a low number of components. Similarly, using the same running data from Reinschmidt et al. (1997), Dumas et al. (2014b) applied a proper orthogonal decomposition and demonstrated that the displacements of the skin markers relative to the underling bone can be represented by a linear combination of a low number of modes. In addition, these main components and modes corresponded to the translation and rotation of the marker-cluster. Therefore, it appeared logical to also associate these components and modes to the wobbling masses vibration. This association between the main modes of the STA and the wobbling masses vibration could be obtained, in the present study, by the smooth orthogonal decomposition. As stated before, this association considers the displacements of the skin markers as a proxy for the wobbling mass movement. As explained by Challis and Pain (2008), "skin-marker motion must be associated with the motion of the underlying soft tissues because skin has low stiffness compared with the stiffness of the muscle-tendon complexes". However, modelling the dynamics of the continuous deformation of the soft tissues (i.e., skin, muscles, and fat) by the discrete movement of a rigid wobbling mass remains challenging. For instance, the proportion of the soft tissues mass involved in the 
wobbling mass is an open question. For a better insight, the analysis of the relative movement between the skin and the bone should be completed by the analysis of the ground reaction forces (not known in the present study). The recent studies which simultaneously track skin markers trajectories and external forces to estimate joint kinematics and segment masses (Bonnet et al., 2017; Jackson et al., 2015) may be adapted to thoroughly identify the parameters of the wobbling mass models. Additionally, the deformable modelling of the lower limb segments (Clark and Hawkins, 2010; Halloran et al., 2010; Stelletta et al., 2017) may help in understanding the dynamics effects of the soft tissue deformation (e.g. mass redistribution, energy dissipation, intra-segmental forces,) and help in verifying if the displacements of the skin markers can be representative of them.

This study had some limitations. First, as mentioned above, the wobbling mass model is only built from the relative movement between the skin and the bone (i.e., STA). Second, the STA vector could be biased by the loosening and bending of the intra-cortical pins (Ramsey and Wretenberg, 1999). Third, this study was based on retrospective data (Benoit et al., 2006; Reinschmidt et al., 1997) and the detailed anthropometry of the three subjects performing the running task as well as the ground reaction forces and muscle activities were not available. Fourth, the study was based on motion capture data (i.e. at $120 \mathrm{~Hz}$ and $200 \mathrm{~Hz}$ ) while accelerometry may be considered more appropriate (Coza et al., 2010). Nevertheless, as the vibration frequencies were in the band $10-30 \mathrm{~Hz}$, the Nyquist-Shannon sample-rate criterion was satisfied. Fifth, the determination of the stiffness matrix using the smooth orthogonal decomposition assumed that the STA field was the results of the free undamped vibrations of a cluster of lumped masses while damping effects are not negligible in the soft tissue vibrations (Khassetarash et al., 2015; Schmitt and Günther, 2011; Wakeling et al., 2003). Nevertheless, benchmark simulations demonstrated that the smooth orthogonal decomposition was also reliable in the case of forced damped vibrations (Chelidze and Zhou, 2006). 
In conclusion, the smooth orthogonal decomposition applied to the measured displacements of the skin markers relative to the underling bone allowed to estimate the stiffness of a wobbling mass model translating about the three axes of the bone-embedded coordinate systems. The estimated stiffness coefficients (i.e. between $2.3 \mathrm{kN} / \mathrm{m}$ and $55.5 \mathrm{kN} / \mathrm{m}$ ) as well as the corresponding forces (i.e. up to $400 \mathrm{~N}$ ) and frequencies (i.e. in the band 10-30 Hz) were in agreement with the literature. The estimated stiffness coefficients were found subject- and task-specific.

\section{Acknowledgements}

The authors would like to thank A. J. van den Bogert of Cleveland State University for kindly providing the unfiltered running data. The authors are also grateful to D. Benoit for having made available the unfiltered walking, cutting and hopping data for this special issue of the Journal of Biomechanics on the soft tissue artefact.

\section{Conflict of interest}

The authors do not have any financial or personal relationships with other people or organizations that would have inappropriately influence this study.

\section{References}

Alonso, F.J., Del Castillo, J.M., Pintado, P., 2007. Motion data processing and wobbling mass modelling in the inverse dynamics of skeletal models. Mechanism and Machine Theory 42, 11531169.

Andersen, M.S., Damsgaard, M., Rasmussen, J., Ramsey, D.K., Benoit, D.L., 2012. A linear soft tissue artefact model for human movement analysis: proof of concept using in vivo data. Gait Posture 35, 606-611.

Bélaise, C., Blache, Y., Thouzé, A., Monnet, T., Begon, M., 2016. Effect of wobbling mass modeling on joint dynamics during human movements with impacts. Multibody System Dynamics 38, 345-366.

Benoit, D.L., Damsgaard, M., Andersen, M.S., 2015. Surface marker cluster translation, rotation, scaling and deformation: Their contribution to soft tissue artefact and impact on knee joint kinematics. Journal of Biomechanics 48, 2124-2129. 
Benoit, D.L., Ramsey, D.K., Lamontagne, M., Xu, L., Wretenberg, P., Renstrom, P., 2006. Effect of skin movement artifact on knee kinematics during gait and cutting motions measured in vivo. Gait Posture 24, 152-164.

Bonnet, V., Dumas, R., Cappozzo, A., Joukov, V., Daune, G., Kulić, D., Fraisse, P., Andary, S., Venture, G., 2017. A constrained extended Kalman filter for the optimal estimate of kinematics and kinetics of a sagittal symmetric exercise. Journal of Biomechanics, In press.

Cerveri, P., Pedotti, A., Ferrigno, G., 2005. Kinematical models to reduce the effect of skin artifacts on marker-based human motion estimation. Journal of Biomechanics 38, 2228-2236.

Challis, J.H., Pain, M.T.G., 2008. Soft Tissue Motion Influences Skeletal Loads During Impacts. Exercise and Sport Sciences Reviews 36, 71-75.

Chelidze, D., Zhou, W., 2006. Smooth orthogonal decomposition-based vibration mode identification. Journal of Sound and Vibration 292, 461-473.

Clark, T., Hawkins, D., 2010. Are fixed limb inertial models valid for dynamic simulations of human movement? Journal of Biomechanics 43, 2695-2701.

Clarys, J.P., Marfell-Jones, M.J., 1986. Anthropometric Prediction of Component Tissue Masses in the Minor Limb Segments of the Human Body. Human Biology 58, 761-769.

Coza, A., Nigg, B.M., Fliri, L., 2010. Quantification of Soft-Tissue Vibrations in Running: Accelerometry versus High-Speed Motion Capture. Journal of Applied Biomechanics 26, 367-372.

Dumas, R., Camomilla, V., Bonci, T., Cheze, L., Cappozzo, A., 2014a. Generalized mathematical representation of the soft tissue artefact. Journal of Biomechanics 47, 476-481.

Dumas, R., Camomilla, V., Bonci, T., Cheze, L., Cappozzo, A., 2014b. A qualitative analysis of soft tissue artefact during running. Computer Methods in Biomechanics and Biomedical Engineering 17, 124-125.

Dumas, R., Camomilla, V., Bonci, T., Chèze, L., Cappozzo, A., 2015. What Portion of the Soft Tissue Artefact Requires Compensation When Estimating Joint Kinematics? Journal of Biomechanical Engineering 137, 064502-064502-064505.

Dumas, R., Cheze, L., Verriest, J.P., 2007. Adjustments to McConville et al. and Young et al. body segment inertial parameters. J Biomech 40, 543-553.

Gittoes, M.J., Kerwin, D.G., Brewin, M.A., 2009. Sensitivity of loading to the timing of joint kinematic strategies in simulated forefoot impact landings. J Appl Biomech 25, 229-237.

Gittoes, M.J.R., Brewin, M.A., Kerwin, D.G., 2006. Soft tissue contributions to impact forces simulated using a four-segment wobbling mass model of forefoot-heel landings. Human Movement Science 25, 775-787.

Grimpampi, E., Camomilla, V., Cereatti, A., de Leva, P., Cappozzo, A., 2014. Metrics for Describing Soft-Tissue Artefact and Its Effect on Pose, Size, and Shape of Marker Clusters. IEEE Transactions on Biomedical Engineering 61, 362-367.

Gruber, K., Ruder, H., Denoth, J., Schneider, K., 1998. A comparative study of impact dynamics: wobbling mass model versus rigid body models. Journal of Biomechanics 31, 439-444.

Günther, M., Sholukha, V.A., Kessler, D., Wank, V., Blickhan, R., 2003. Dealing with skin motion and wobbling masses in inverse dynamics. Journal of Mechanics in Medicine and Biology 03, 309-335.

Halloran, J.P., Ackermann, M., Erdemir, A., van den Bogert, A.J., 2010. Concurrent musculoskeletal dynamics and finite element analysis predicts altered gait patterns to reduce foot tissue loading. Journal of Biomechanics 43, 2810-2815.

Jackson, J.N., Hass, C.J., Fregly, B.J., 2015. Residual Elimination Algorithm Enhancements to Improve Foot Motion Tracking During Forward Dynamic Simulations of Gait. Journal of Biomechanical Engineering 137, 111002-111002-111008.

Khassetarash, A., Hassannejad, R., Enders, H., Ettefagh, M.M., 2015. Damping and energy dissipation in soft tissue vibrations during running. Journal of Biomechanics 48, 204-209.

Lafortune, M.A., Cavanagh, P.R., Sommer lii, H.J., Kalenak, A., 1992. Three-dimensional kinematics of the human knee during walking. Journal of Biomechanics 25, 347-357.

Lafortune, M.A., Henning, E., Valiant, G.A., 1995. Tibial shock measured with bone and skin mounted transducers. Journal of Biomechanics 28, 989-993. 
Leardini, A., Chiari, L., Della Croce, U., Cappozzo, A., 2005. Human movement analysis using stereophotogrammetry. Part 3. Soft tissue artifact assessment and compensation. Gait Posture 21, 212-225.

McLean, S.G., Su, A., van den Bogert, A.J., 2003. Development and Validation of a 3-D Model to Predict Knee Joint Loading During Dynamic Movement. Journal of Biomechanical Engineering 125, 864-874.

Nigg, B.M., Wakeling, J.M., 2001. Impact Forces and Muscle Tuning: A New Paradigm. Exercise and Sport Sciences Reviews 29, 37-41.

Pain, M.T.G., Challis, J.H., 2004. Wobbling Mass Influence on Impact Ground Reaction Forces: A Simulation Model Sensitivity Analysis. Journal of Applied Biomechanics 20, 309-316.

Pain, M.T.G., Challis, J.H., 2006. The influence of soft tissue movement on ground reaction forces, joint torques and joint reaction forces in drop landings. Journal of Biomechanics 39, 119-124.

Peters, A., Galna, B., Sangeux, M., Morris, M., Baker, R., 2010. Quantification of soft tissue artifact in lower limb human motion analysis: A systematic review. Gait \& Posture 31, 1-8.

Ramsey, D.K., Wretenberg, P.F., 1999. Biomechanics of the knee: methodological considerations in the in vivo kinematic analysis of the tibiofemoral and patellofemoral joint. Clinical Biomechanics 14, 595-611.

Reinschmidt, C., van den Bogert, A.J., Nigg, B.M., Lundberg, A., Murphy, N., 1997. Effect of skin movement on the analysis of skeletal knee joint motion during running. Journal of Biomechanics 30 , 729-732.

Richard, V., Camomilla, V., Cheze, L., Cappozzo, A., Dumas, R., 2012. Feasibility of incorporating a soft tissue artefact model in multi-body optimisation. Computer Methods in Biomechanics and Biomedical Engineering 15, 194-196.

Schmitt, S., Günther, M., 2011. Human leg impact: energy dissipation of wobbling masses. Archive of Applied Mechanics 81, 887-897.

Stelletta, J., Dumas, R., Lafon, Y., 2017. Modeling of the thigh: a 3D deformable approach considering muscle interactions, in: Payan, Y., Ohayon, J. (Eds.), Biomechanics of living organs: hyperelastic constitutive laws for finite element modeling. Elsevier.

Wakeling, J.M., Liphardt, A.-M., Nigg, B.M., 2003. Muscle activity reduces soft-tissue resonance at heel-strike during walking. Journal of Biomechanics 36, 1761-1769.

Wilson, C., King, M.A., Yeadon, M.R., 2006. Determination of subject-specific model parameters for visco-elastic elements. Journal of Biomechanics 39, 1883-1890. 


\section{Table and figure captions}

Table 1: Subjects' demographics and experimental conditions

Figure 1: Boxplot for the stiffness coefficients about the $\mathrm{X}, \mathrm{Y}$ and $\mathrm{Z}$ axes of the boneembedded coordinate systems for the different subjects and tasks

Figure 2: Forces about the $\mathrm{X}, \mathrm{Y}$, and $\mathrm{Z}$ axes of the bone-embedded coordinate systems for the different subjects and motor tasks 
Table 1

\begin{tabular}{|c|c|c|c|c|c|c|c|c|c|}
\hline \multirow[b]{2}{*}{ Subject } & \multirow[b]{2}{*}{$\begin{array}{c}\text { Age } \\
\text { (years) }\end{array}$} & \multirow[b]{2}{*}{$\begin{array}{c}\text { Mass } \\
(\mathrm{kg})\end{array}$} & \multirow[b]{2}{*}{$\begin{array}{l}\text { Height } \\
\text { (m) }\end{array}$} & \multirow[b]{2}{*}{ Motor task } & \multirow[b]{2}{*}{$\begin{array}{c}\mathrm{f} \\
(\mathrm{Hz})\end{array}$} & \multicolumn{2}{|c|}{ Shank $(i=1)$} & \multicolumn{2}{|c|}{ Thigh $(i=2)$} \\
\hline & & & & & & $m_{1}$ & $\begin{array}{l}M_{1} * \\
(\mathrm{~kg})\end{array}$ & $m_{2}$ & $\begin{array}{l}M_{2} \# \\
(\mathrm{~kg})\end{array}$ \\
\hline $\begin{array}{l}\mathrm{R} 1, \mathrm{R} 2, \\
\text { and R3 }\end{array}$ & $25.7 \pm 2.1$ & $85.5 \pm 9.6$ & $186.7 \pm 9.6$ & Running & 200 & 6 & 3.19 & & 9.50 \\
\hline B1 & 22 & 63 & 175 & $\begin{array}{l}\text { Walking, } \\
\text { cutting, and } \\
\text { hopping }\end{array}$ & 120 & 4 & 2.35 & 4 & 7.00 \\
\hline \multicolumn{10}{|c|}{$*(4.8 \times 77.65) \%$ of averaged or subject's mass } \\
\hline$\#(12.3 \times$ & 3) $\%$ of av & ged or sub & mass & & & & & & \\
\hline
\end{tabular}


Figure 1
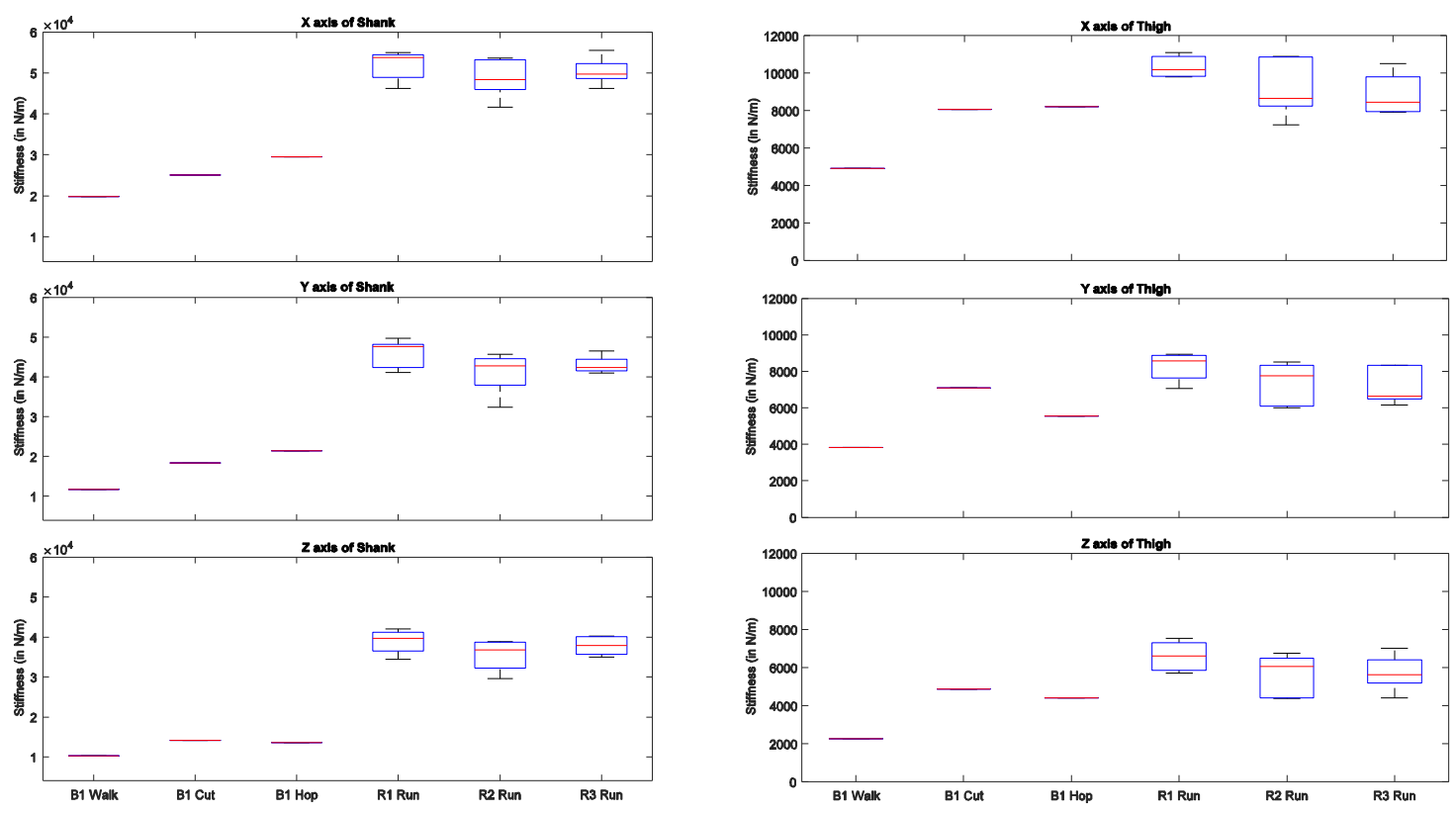
Figure 2
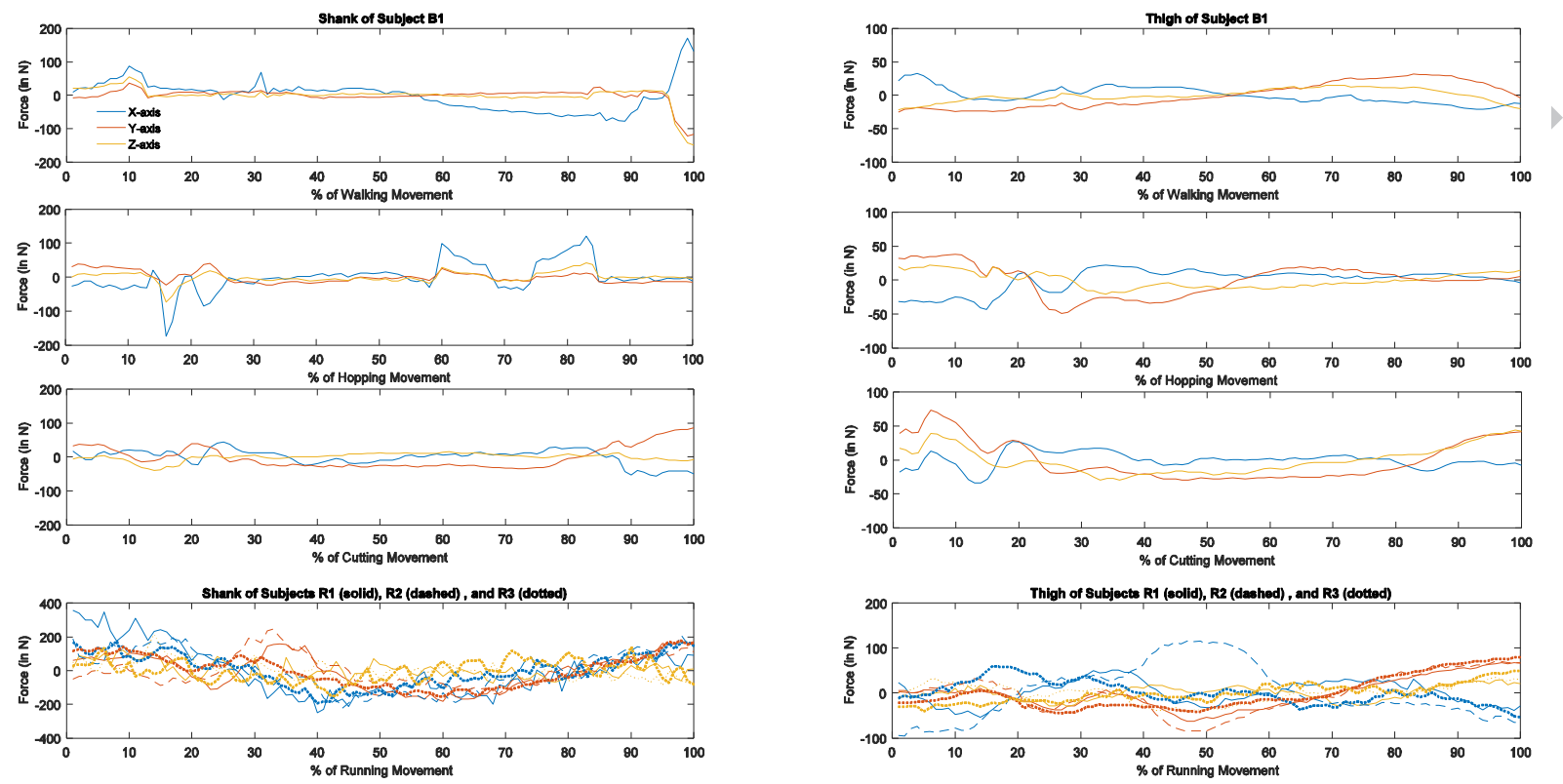